\title{
Hiperplasia de cóndilo mandibular: Reporte de doce casos
}

\section{Hyperplasia of the mandibular condyle: Considerations in a series of 12 patients}

\author{
Dahiana Pulgar B', Ignacio Goñi E, Carmen Reinoso D², Rolando Schulz R², Fernando Hormazábal N³, \\ Alex Vargas D¹, Hernán Ramírez S'1.
}

\begin{abstract}
RESUMEN
Introducción: La hiperplasia condilar (HC) se define como un crecimiento patológico no neoplásico que afecta tanto al tamaño como a la morfología del cóndilo mandibular.

Objetivo: Evaluar la eficacia y riesgos del tratamiento quirúrgico de la $\mathrm{HC}$.

Material y método: Estudio descriptivo, serie de casos. Se incluyó a todos los pacientes con diagnóstico de HC tratados en el Hospital Clínico de la Pontificia Universidad Católica de Chile entre enero de 2010 y febrero de 2014.

Resultados: Doce pacientes fueron operados en nuestra institución por HC. El promedio de edad fue 19,3 \pm 3,4 años. La forma de presentación más frecuente fue presencia de asimetría facial. Todos los pacientes fueron estudiados con SPECT que evidenció diferencia de captación mayor a 50\% $\pm 3,1 \%$ entre ambos cóndilos. Todos los pacientes fueron tratados con condilectomía mandibular alta del lado afectado y en 3 casos, se realizó además cirugía ortognática en el mismo tiempo para corrección de maloclusión dental. La mediana de seguimiento fue de meses 16 (5 a 54 meses). En todos los pacientes se evidenció una mejora en la oclusión dental y simetría facial, con desaparición de la disfunción articular previa.

Conclusiones: La HC es una entidad de poca frecuencia. La cirugía es eficaz y segura para el tratamiento de la $\mathrm{HC}$.

Palabras clave: Hiperplasia condilar, condilectomía mandibular, trastornos temporomandibulares.
\end{abstract}

\begin{abstract}
Introduction: Condylar hiperplasia is defined as a pathological non neoplastic growth which compromises both size and shape of the mandibular condyle and is characterized by progressive facial asymmetry.

2 Unidad de Ortopedia Dentomaxilar y Ortodoncia, Centro Médico San Joaquín, Pontificia Universidad Católica de Chile.

3 Unidad de Trastornos Témporo Mandibulares y Dolor Orofacial, Centro Médico San Joaquín, Pontificia Universidad Católica
\end{abstract}

Departamento de Cirugía Oncológica y Maxilofacial, Pontificia Universidad Católica de Chile. de Chile. 
Aim: To evaluate the efficacy and risks of surgical treatment of HC.

Material and method: Descriptive study, cohort of cases. All patients between 2010 and February 2014 with diagnosis of condylar hyperplasia at Hospital Clínico P. Universidad Católica de Chile were included.

Results: Twelve patients were operated at our institution because of condylar hyperplasia. Age average was $19.3 \pm 3.4$ years. The most frequent presentation was facial asymmetry. All patients were studied with single photon emission computed tomography (SPECT) with differential intake more than $50 \pm 3.1 \%$ between both condyles. All patients were treated with mandibular condylectomy of the affected site and in 3 cases orthognatic surgery was performed at the same time of the condylectomy to correct dental malocclusion. Average follow up was 16 months (5 to 54 months). All patients recovered facial symmetry and occlusion with absence of the previous joint dysfunction.

Conclusions: Condylar hyperplasia is an unfrequent condition and surgery is efficient and safe for the treatment of the deformity.

Key words: Condylar hyperplasia, mandibular condylectomy, temporomandibular disorders.

\section{INTRODUCCIÓN}

La hiperplasia condilar (HC) fue descrita por primera vez por Robert Adams el año 1836'. Se define como un crecimiento anormal no neoplásico del cóndilo mandibular que afecta consecutivamente la simetría facial y oclusal pudiendo generar dolor y disfunción en la articulación temporomandibular (ATM) habitualmente del lado sano ${ }^{2,3}$. Se caracteriza por ser generalmente unilateral y comportarse como una patología progresiva, deformante y no siempre autolimitada, con predominio en la segunda y tercera década de vida ${ }^{4}$. A pesar de existir controversia en la literatura, se acepta que esta patología posee una predilección por el género femenino y presenta una distribución similar en raza y lado comprometido $0^{2,5}$

La etiología de la HC aún se mantiene incierta. Se discuten una serie de elementos que pueden participar activamente como factores causales de HC, entre ellos: proliferación excesiva posterior a trauma 0 infecciones, influencia genética, desórdenes endocrinos, factores funcionales 10cales, enfermedades degenerativas de la ATM, alteraciones en el aporte sanguíneo y hábitos parafuncionales, los cuales pueden determinar el crecimiento excesivo del cóndilo mandibular-9.9.

Las deformidades que causa esta alteración son muy variadas tanto en características clínicas como en severidad. La HC ha sido clasificada en varias ocasiones y según distintos criterios.
Norman y Painter ${ }^{5}$, categorizan la $\mathrm{HC}$ en activa 0 inactiva según la progresión de ésta o la comprobación de una actividad ósea metabólica alterada. Obwegeser y Makek ${ }^{10}$ la clasifican, según el patrón de crecimiento que predomine en la estructura afectada en: tipo 1 (vector de crecimiento horizontal), tipo 2 (vector de crecimiento vertical) y una presentación mixta.

Los signos y síntomas principales de esta patología, son la asimetría facial y mandibular, alteraciones oclusales con mordida abierta ipsilateral y/o cruzada contralateral, compensaciones óseas y dentales con elongación, y laterognasia y/o pseudoprognatismo asimétrico asociado $0^{3,10}$. Además, puede aparecer dolor capsular y retrodiscal, luxaciones disco-condilares y limitación en la dinámica mandibular, muchas veces como cambios reactivos a la hiperfunción en el lado sano ${ }^{10}$.

El diagnóstico de HC se realiza por la presencia de manifestaciones clínicas sumadas a la imagenología. Comúnmente se utiliza a radiografía panorámica y la tomografía con reconstrucción 3D para evidenciar la presencia de un cóndilo hiperplásico y de asimetría facial ${ }^{11}$. Para determinar la actividad metabólica aumentada en el cóndilo afectado se utiliza frecuentemente una cintigrafía ósea con tecnecio 99, un PET (tomografía por emisión de positrones) 0 un SPECT (tomografía por emisión de fotón único) ${ }^{12}$. El SPECT es método diagnóstico de elección para la HC ya que permite cuantificar y comparar la actividad osteoblástica en 
las ATM derecha e izquierda. Kajan y $\operatorname{cols}^{13}$, en un estudio que incluyó a 38 pacientes sin hallazgos clínicos ni radiográficos de $\mathrm{HC}$ demostró que un índice de captación relativo entre la ATM derecha/ izquierda es normal si la diferencia porcentual es hasta de 6,2\% (50 $\pm 3,1 \%)$ entre ambos cóndilos. Se reporta en la literatura una sensibilidad del SPECT para la detección HC del $90 \%$ (78\% a $97 \%)$ comparada con $71 \%$ (57\% a $82 \%)$ de la cintigrafía ósea planar ${ }^{12}$, no existiendo diferencia en la especificidad de ambos métodos ${ }^{12}$. Sin embargo, dada la ausencia de un gold standard para el diagnóstico de $\mathrm{HC}$, el examen clínico continúa siendo fundamental, ya que un cóndilo contralateral inflamado 0 en remodelación por sobrecarga puede presentarse con una mayor actividad cintigráfica, lo que constituye un falso negativo ${ }^{14}$.

Diversas alternativas para el tratamiento de pacientes con HC han sido descritas en la literatura, las que varían según distintos parámetros como son la confirmación de crecimiento condilar activo, la severidad de la deformación dento-facial existente, la presencia de una deformidad preexistente, la edad del individuo y la sintomatología 0 presencia de disfunción articular ${ }^{15,16}$.

\section{OBJETIVO}

Existen pocos reportes nacionales sobre el manejo de la $\mathrm{HC}^{17}$. Nuestro objetivo primario es evaluar la eficacia y riesgos del tratamiento quirúrgico de la HC. Los objetivos específicos son describir las características demográficas, estudio preoperatorio, tratamiento quirúrgico y seguimiento a largo plazo.

\section{MATERIAL Y MÉTODO}

\section{Diseño: Descriptivo. Serie de casos.}

Pacientes: Se incluyó a todos los pacientes con diagnóstico de $\mathrm{HC}$ ingresados en el policlínico de cirugía maxilofacial del Centro Médico San Joaquín de la Pontificia Universidad Católica de Chile que recibieron tratamiento quirúrgico entre enero de 2010 y febrero de 2014. Se realizó evaluación interdisciplinaria a todos los pacientes por cirugía maxilofacial, ortodoncia y especialidad en trastornos temporomandibulares, realizándose examen clínico e imagenológico. El diagnóstico de $\mathrm{HC}$ se realizó con una clínica compatible y la presencia de un SPECT que mostrara diferencia de captación condilar $>6,2 \%$ sin evidencias de inflamación actual a nivel de la articulación témporomadibular.

Fuentes: Los datos se recolectaron de los registros de fichas clínicas y registros de biopsias de nuestro centro. Para el seguimiento se obtuvo información desde los registros clínicos.

Variables: Las variables consideradas fueron: sexo, edad, comorbilidades, antecedentes familiares de HC, presentación clínica, exámenes de laboratorio, estudio imagenológico, tipo de cirugía realizada, morbilidad posoperatorio, anatomía patológica posoperatoria, exámenes de laboratorio posoperatorios, recidiva y reintervenciones.

Análisis estadístico: Para el análisis estadístico se utilizó programa SPSS 15. Se realizó estadística descriptiva. Para comparar variables, se realizó pruebas de hipótesis paramétricas y no paramétricas según la determinación de normalidad. Se consideró estadísticamente significativo un nivel de confianza del $95 \%$ con un valor $p<0,05$.

\section{RESULTADOS}

En el periodo descrito, 12 pacientes fueron operados en nuestra institución por HC. El promedio de edad fue 19,3 $\pm 3,4$ años. Ocho casos $(66,7 \%)$ correspondieron a sexo femenino y $4(33,3 \%)$ a sexo masculino. La Tabla 1 resume las principales características clínicas de los pacientes.

\section{Presentación clínica}

El motivo de consulta comprendió la presencia de asimetría facial progresiva en 7 pacientes $(58,4 \%)$, alteraciones en articulación temporomandibular (clicking y dolor) en 1 paciente $(8,3 \%)$ y en 4 pacientes la combinación de ambos (33,3\%).

Al examen físico: Todos los pacientes presentaron un crecimiento excesivo unilateral del cóndilo mandibular con alteración de la oclusión dental 
Tabla 1. Características de los pacientes con diagnóstico de HC ( $\mathrm{n}=12)$

\begin{tabular}{|c|c|c|}
\hline \multicolumn{2}{|c|}{ Variable } & $N^{\circ}$ pacientes \\
\hline Sexo & $\begin{array}{l}\text { Femenino } \\
\text { Masculino }\end{array}$ & $\begin{array}{l}8 \\
4\end{array}$ \\
\hline Edad & $\begin{array}{l}\leq 20 \text { años } \\
>20 \text { años }\end{array}$ & $\begin{array}{l}7 \\
5\end{array}$ \\
\hline Motivo de consulta & $\begin{array}{l}\text { Asimetría facial } \\
\text { Síntomas ATM } \\
\text { Presencia de ambas }\end{array}$ & $\begin{array}{l}7 \\
1 \\
4\end{array}$ \\
\hline Lado afectado & $\begin{array}{l}\text { Derecho } \\
\text { Izquierdo }\end{array}$ & $\begin{array}{l}7 \\
5\end{array}$ \\
\hline Clasificación de Ob & $\begin{array}{l}\text { vegeser y Makek } \\
\text { Tipo I } \\
\text { Tipo II } \\
\text { Combinación ambas }\end{array}$ & \\
\hline
\end{tabular}

concomitante y/o la desviación del mentón hacia el lado opuesto (Figura 1). Según la clasificación clínica de Obwegeser y Makek ${ }^{10}, 4$ pacientes fueron considerados tipo $1(\%), 6$ pacientes tipo $2(\%)$, y una combinación de ambos tipos se observó en 2 pacientes (\%).

\section{Evaluación preoperatoria}

A todos los pacientes se les realizó estudio imagenológico con ortopantomografía, telerradiografía frontal y lateral de cráneo con cefalometrías y SPECT óseo (Figura 2). En todos los casos, se observó una asimetría en la absorción del radioisótopo (Tecnecio 99) a nivel condilar. La máxima diferencia de captación cuantificada entre ambos cóndilos fue de $59,9 \%$ y $40 \%$, en el lado hiperplásico y normal respectivamente. El promedio de captación de Tecnecio 99 del cóndilo afectado fue 57,3\% ( \pm $1,93)$, mientras que el del cóndilo sano fue de $42,7 \%( \pm 1,83)$.

\section{Tratamiento quirúrgico}

El tipo de cirugía realizada varió según el grado de severidad de la HC y de la deformidad dentoesquelética asociada. En todos los pacientes se realizó condilectomía alta del lado afectado (Figura $3)$. En tres casos (25\%) se asoció la condilectomía a cirugía ortognática en el mismo tiempo quirúrgico para corrección de la maloclusión dental.

La mediana de días de hospitalización fue de 2 días (1 a 6 días). La complicación posoperatoria más frecuente fue la presencia de paresia transitoria de la rama orbitaria nervio del facial en 5 casos (42\%). Dos pacientes presentaron infección de herida operatoria siendo manejados con antibioterapia oral en forma ambulatoria. El tratamiento quirúrgico y resultados posoperatorios de los pacientes se detallan en la Tabla 2.

\section{Seguimiento a largo plazo}

La mediana de seguimiento fue de meses 16 ( 5 a 54 meses). En todos los pacientes se evidenció una mejora en la oclusión dental y simetría facial, con desaparición de la sintomatología articular previa
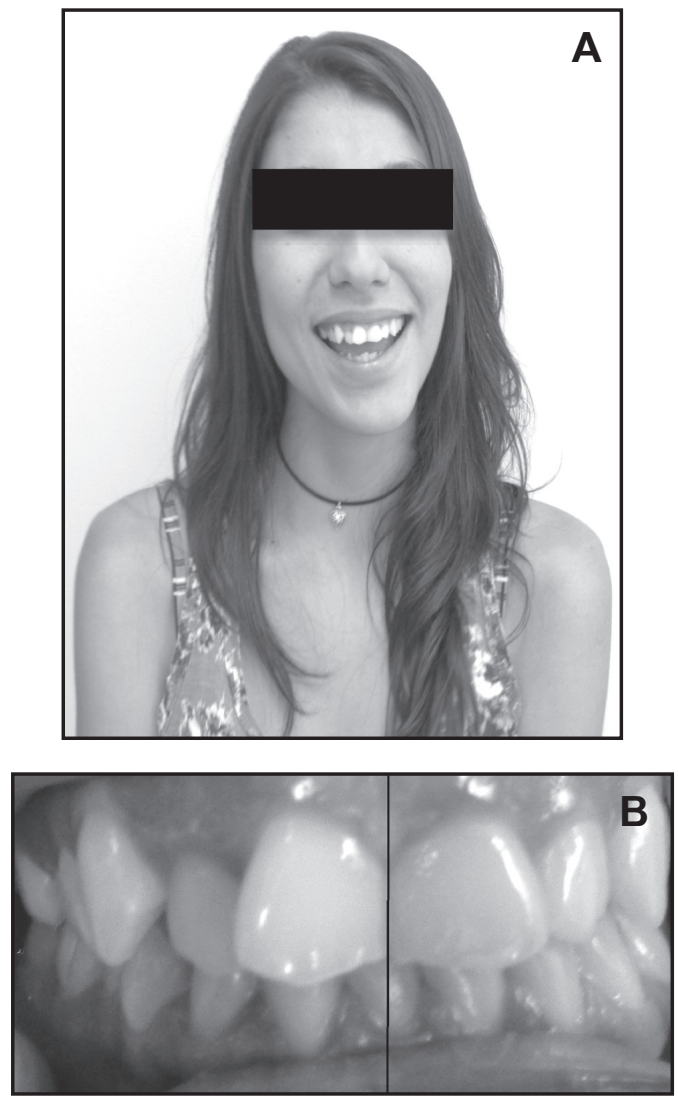

Figura 1. Hallazgos al examen físico de pacientes con HC. A. Asimetría facial. B. Desviación de la línea media dentaria arcada inferior. 

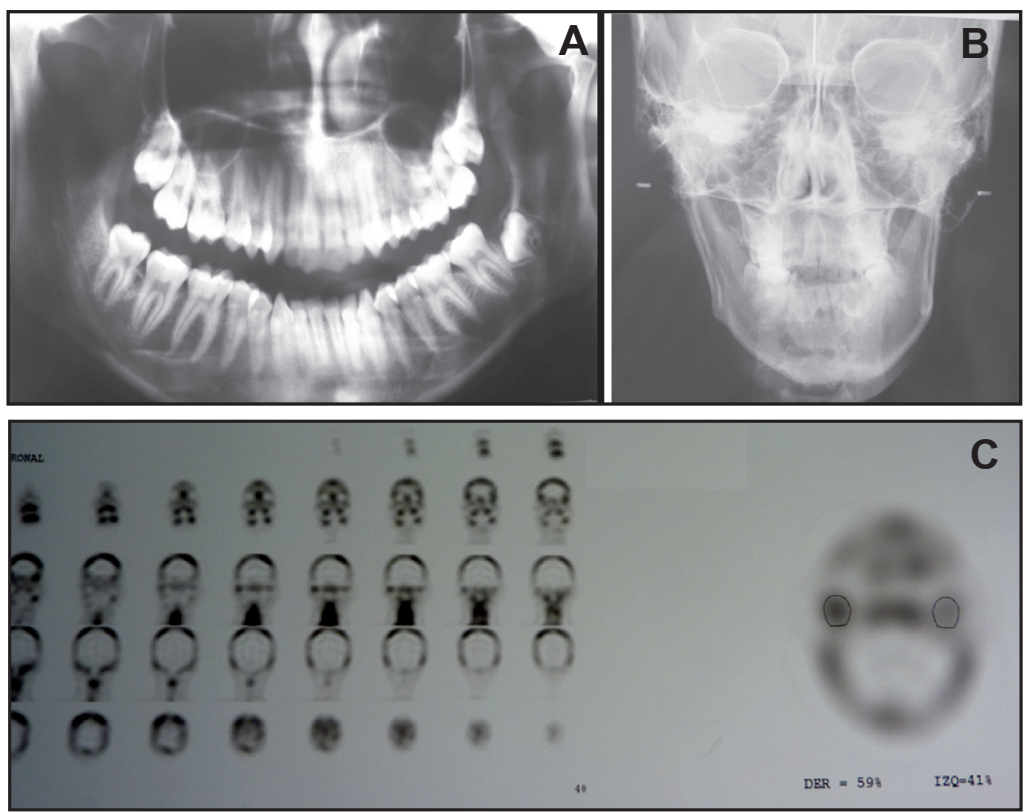

Figura 2. Hallazgos en el estudio imagenológico de pacientes con HC. A. Radiografía panorámica, elongación vertical del cóndilo derecho. B. Radiografía con proyección AP, asimetría facial por mayor crecimiento de cóndilo derecho. C. SPECT con diferencia en captación condilar >6,2\% (der: $59 \%$ vs izq: $41 \%$ ).

Tabla 2. Tratamiento quirúrgico y resultados posoperatorios de pacientes con HC $(n=12)$

\begin{tabular}{|l|c|}
\hline \multicolumn{1}{|c|}{ Variable } & $\mathrm{N}^{\circ}$ de pacientes \\
\hline Tipo de cirugía & \\
\hline - Condilectomía mandibular & 9 \\
\hline - Cirugía ortognática asociada & 3 \\
\hline Complicaciones posoperatorias & \\
\hline - Paresia nervio facial & 5 \\
\hline - Infección herida operatoria & 2 \\
\hline - Hipoestesia zona pretragal & 1 \\
\hline - Hipometría oral & 1 \\
\hline
\end{tabular}

en todos los casos. No se evidenció recurrencia en esta serie de pacientes durante el seguimiento.

\section{DISCUSIÓN}

La hiperplasia condilar es una entidad poco frecuente caracterizada por un crecimiento patológico no neoplásico que afecta tanto al tamaño como a la morfología del cóndilo mandibular. Si bien la etiología es desconocida, se reconoce que es la zona de osificación subcondral del cóndilo mandibular el tejido que reacciona a un estímulo desconocido 2,10 . Se ha planteado además una posible influencia

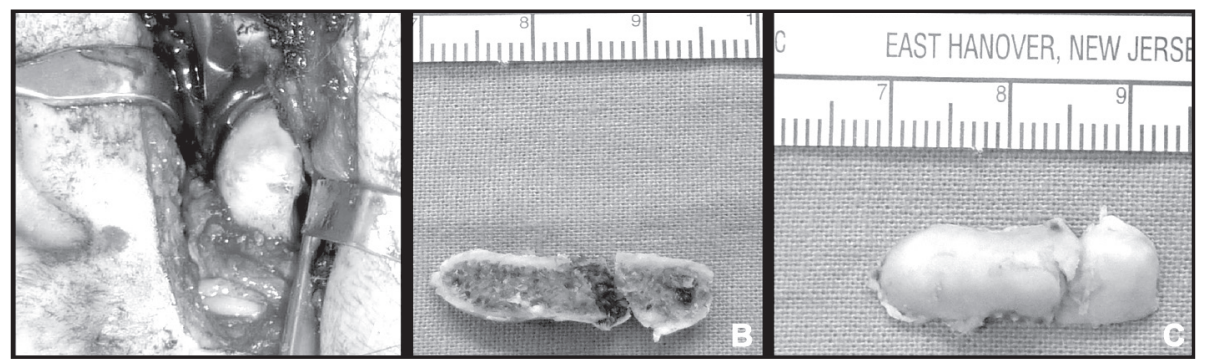

Figura 3. Imágenes del tratamiento quirúrgico de pacientes con HC. A. Abordaje de Al-Kayat para la articulación temporomandibular. B y C Pieza operatoria de condilectomía alta, evidenciando osteotomía de cabeza condilar hipertrófica, en segmento de $5 \mathrm{~mm}$ aproximadamente de altura. 
genética en algunos casos en los cuales se podría encontrar una herencia ligada al cromosoma $Y_{0}$ autosómica dominante ${ }^{9}$; sin embargo, se necesitan series con mayor número de casos para respaldar esta teoría.

La HC se presenta clínicamente con asimetría facial, alteraciones en la oclusión dental y disfunción de la articulación temporomandibular. Se manifiesta de manera predominante en adolescentes durante el período de crecimiento y en adultos jóvenes que ya han terminado su crecimiento, no existe predilección en cuanto a raza ni lado comprometido. En nuestra serie, la HC fue más frecuente en el sexo femenino, lo que es compatible con algunos reportes de la literatura ${ }^{2,18}$. Diversos estudios, han reportado un aumento en el número de receptores de estrógeno en la articulación temporomandibular en las mujeres lo que podría ser la causa del incremento en la actividad de crecimiento del cóndilo mandibular y del predominio de la $\mathrm{HC}$ en el sexo femenino ${ }^{19,20}$.

Las alteraciones esqueléticas asociadas a la HC son múltiples y de severidad variable, esto se debe a que el crecimiento mandibular ocurre en los tres planos del espacio (en sentido horizontal, vertical y transversal) pero con predominio de alguno de ellos ${ }^{10}$. En nuestros pacientes, según la clasificación clínica de Obwegeser y Makek ${ }^{10}$, el tipo más frecuente correspondió al tipo 2 con 6 casos, lo que es concordante con lo reportado en la literatura ${ }^{2,21}$.

No existe un gold standard para el diagnóstico de hiperplasia condilar, siendo más bien el conjunto de hallazgos clínicos, imagenológicos y funcionales la clave para la identificación de la enfermedad. Sin embargo, se considera al SPECT como el examen de elección para la evaluación de la HC. Todos los métodos de SPECT disponibles en la actualidad para el diagnóstico de HC utilizan métodos de cuantificación basados en la diferencia de captación de radioisótopo entre ambos cóndilos. En nuestra serie se consideró un SPECT con diferencia de captación condilar $>6,2 \%$ asociado a clínica compatible como diagnóstico de HC. En la literatura diversas series consideran normal una diferencia de captación condilar menor de $10 \%$ por SPECT. Esta diferencia se debe a que la forma para calcular la captación diferencial a nivel de cóndilos mandibulares no está claramente estandarizada.
No existe un enfoque terapéutico único para el manejo de esta heterogénea condición maxilofacial puesto que no existe una deformidad usual. A pesar de esto, se proponen una serie de esquemas terapéuticos, principalmente ortodóncico-quirúrgicos, en los cuales se revierten las compensaciones y se llevan las piezas dentarias a sus bases óseas, creando arcos dentales armónicos en posición de ventaja, tal como se hace en cirugía ortognática ${ }^{4,22}$. En caso de sintomatología articular, se instaura un tratamiento ortopédico tendiente a aliviar la sobrecarga funcional articular y a determinar la posición céntrica mandibular estable para el posoperatorio ${ }^{23}$.

La cirugía se enfoca primariamente a eliminar la zona de crecimiento activo mediante una condilectomía alta, en general de 3 a $5 \mathrm{~mm}$, que compromete toda la superficie de la cabeza condilar ${ }^{4,24}$. Este puede ser un procedimiento único cuando la ostectomía del cóndilo es suficiente para restituir la línea media y la armonía de los arcos dentarios ${ }^{4}$. En nuestros pacientes, en 9 casos se realizó solo condilectomía alta para el manejo de la $\mathrm{HC}$ y en 3 pacientes se realizó en forma asociada a cirugía ortognática en el mismo acto quirúrgico. La cirugía ortognática se realiza en forma asociada cuando el paciente está preparado ortodóncicamente y habitualmente consiste en una osteotomía sagital de rama bilateral o contralateral, a veces acompañada de osteotomía maxilar completa y genioplastía para llevar a posición y corregir las estructuras esqueletales deformadas por el excesivo crecimiento condilar ${ }^{4}$.

Dentro de la morbilidad asociada al tratamiento quirúrgico de la $\mathrm{HC}$, la paresia del ramo frontral del nervio facial es la más frecuentemente descrita ${ }^{21,24}$. En nuestra serie, se privilegió la condilectomía alta usando el abordaje descrito por Al Kayat ${ }^{25}$ para lograr una adecuada preservación del nervio facial, pese a ello, el $42 \%$ de pacientes desarrolló esta complicación en forma transitoria.

\section{CONCLUSIÓN}

El manejo y evaluación de pacientes con HD requiere de un equipo multidisciplinario donde participen cirujanos maxilofaciales, ortodoncistas y especialistas en trastornos temporomandibulares. 
La cirugía es eficaz y segura para el tratamiento de pacientes con HC siendo la condilectomía alta acompañada de discopexia el tratamiento de elección. La cirugía ortognática no es necesaria en todos los pacientes y puede realizarse en el mismo tiempo de la condilectomía o en forma diferida.

\section{BIBLIOGRAFÍA}

1. Rushton MA. Unilateral hyperplasia of the mandibular condyle. Proceedings of the Royal Society of Medicine 1946; 39: 431-8.

2. Nitzan DW, Katsnelson A, Bermanis I, Brin I, CASAP N. The clinical characteristics of condylar hyperplasia: experience with 61 patients. Journal of oral and maxillofacial surgery: Official Journal of the American Association of Oral and Maxillofacial Surgeons 2008; 66(2): 312-8.

3. Olate S, Netto HD, Rodríguez-Chessa J, Alister JP, de Albergaria-Barbosa J, de Moraes M. Mandible condylar hyperplasia: a review of diagnosis and treatment protocol. International Journal of Clinical and Experimental Medicine 2013; 6(9): 727-37.

4. Villanueva-Alcojol L, Monje F, González-García R. Hyperplasia of the mandibular condyle: clinical, histopathologic, and treatment considerations in a series of 36 patients. Journal of oral and maxillofacial surgery: Official Journal of the American Association of Oral and Maxillofacial Surgeons 2011; 69(2): 447-55.

5. Norman JE, Painter DM. Hyperplasia of the mandibular condyle. A historical review of important early cases with a presentation and analysis of twelve patients. Journal of Maxillofacial Surgery 1980; 8(3): 161-75.

6. Gray RJ, Sloan P, Quayle Aa, Carter DH. Histopathological and scintigraphic features of condylar hyperplasia. International Journal of Oral and Maxillofacial Surgery 1990; 19(2): 65-71.

7. Eslami B, Behnia $H$, Javadi $H$, Khiabani KS, Saffar AS. Histopathologic comparison of normal and hyperplastic condyles. Oral surgery, oral medicine, oral pathology, oral radiology and endodontics 2003; 96(6): 711-7.

8. EGYEDI P. Aetiology of condylar hyperplasia. Australian Dental Journal 1969; 14(1): $12-7$.
9. Yang J, Lignelli JL, Ruprecht A. Mirror image condylar hyperplasia in two siblings. Oral surgery, oral medicine, oral pathology, oral radiology, and endodontics 2004; 97(2): 281-5.

10. Obwegeser HL, Makek MS. Hemimandibular hyperplasia--hemimandibular elongation. Journal of Maxillofacial Surgery 1986; 14(4): 183-208.

11. Мutoh Y, Ohashi Y, Uchiyama N, Terada K, Hanada K, SASAKI F. Three-dimensional analysis of condylar hyperplasia with computed tomography. Journal of cranio-maxillo-facial surgery: Official Publication of the European Association for Cranio-Maxillo-Facial Surgery 1991; 19(2): 4955.

12. Saridin CP, Raijmakers PG, Tuinzing DB, Becking AG. Bone scintigraphy as a diagnostic method in unilateral hyperactivity of the mandibular condyles: a review and meta-analysis of the literature. International Journal of Oral and Maxillofacial Surgery 2011; 40(1): 11-7.

13. Kajan ZD, Motevasseli S, Nasab NK, Ghanepour $H$, ABBASPUR F. Assessment of growth activity in the mandibular condyles by single-photon emission computed tomography (SPECT). Australian Orthodontic Journal 2006; 22(2): 127-30.

14. Derlin T, Busch JD, Habermann CR. 99mTcMDP SPECT/CT for assessment of condylar hyperplasia. Clinical Nuclear Medicine 2013; 38(1): e48-9.

15. Lippold C, Kruse-Losler B, Danesh G, Joos U, Meyer U. Treatment of hemimandibular hyperplasia: the biological basis of condylectomy. The British Journal of Oral \& Maxillofacial Surgery 2007; 45(5): 353-60.

16. JONES RH, TIER GA. Correction of facial asymmetry as a result of unilateral condylar hyperplasia. Journal of oral and maxillofacial surgery: Official Journal of the American Association of Oral and Maxillofacial Surgeons 2012; 70(6): 1413-25.

17. Olate S, De Moraes M. Deformidad Facial Asimétrica: Papel de la Hiperplasia Condilar. Int J Odontostomat 2012; 6 n.3: 337-47.

18. Slootweg PJ, Muller H. Condylar hyperplasia. A clinico-pathological analysis of 22 cases. Journal of Maxillofacial Surgery 1986; 14(4): 209-14.

19. Raijmakers PG, Karssemakers LH, Tuinzing DB. Female predominance and effect of gender on unilateral condylar hyperplasia: a review and 
meta-analysis. Journal of Oral and Maxillofacial Surgery: Official Journal of the American Association of Oral and Maxillofacial Surgeons 2012; 70(1): e72-6.

20. Ribeiro-Dasilva MC, Peres Line SR, Leme Godoy dos Santos MC, Arthuri MT, Hou W, Fillingim RB, Y cols. Estrogen receptor-alpha polymorphisms and predisposition to TMJ disorder. The Journal of Pain: Official Journal of the American Pain Society 2009; 10(5): 527-33.

21. Alyamani A, Abuzinada S. Management of patients with condylar hyperplasia: A diverse experience with 18 patients. Annals of Maxillofacial Surgery 2012; 2(1): 17-23.

22. Avelar RL, Becker OE, Dolzan Ado N, Goelzer JG, Haas OL, JR., DE OLIVEIRA RB. Correction of facial asymmetry resulting from hemimandibular hyperplasia: surgical steps to the esthetic result. The Journal of Craniofacial Surgery 2012; 23(6): 1898-900.
23. Mazzone N, Matteini C, Incisivo V, Belli E. Temporomandibular joint disorders and maxillomandibular malformations: role of condylar "repositionin" plate. The Journal of Craniofacial Surgery 2009; 20(3): 909-15.

24. Wolford LM, Mehra P, Reiche-Fischel 0, MoralesRyan CA, Garcia-Morales P. Efficacy of high condylectomy for management of condylar hyperplasia. American Journal of Orthodontics and Dentofacial Orthopedics: Official Publication of the American Association of Orthodontists, its Constituent Societies, and the American Board of Orthodontics 2002; 121(2): 136-50; discussion 50-1.

25. Al-Kayat A, Bramley P. A modified pre-auricular approach to the temporomandibular joint and malar arch. The British Journal of Oral Surgery 1979; 17(2): 91-103.

Dirección: Hernán Ramírez S.

Departamento de Cirugía Oncológica y Maxilofacial, Pontificia Universidad Católica de Chile Marcoleta 352, Santiago, Chile

E mail: hramirez.skinner@gmail.com 


\title{
Evolución en la calidad de la voz en pacientes disfónicos del Hospital de La Serena tratados con terapia vocal
}

\author{
Changes in the quality of voice in dysphonic patients in the \\ La Serena Hospital Treated with vocal therapy
}

\author{
Carolina Martínez S1.
}

\begin{abstract}
RESUMEN
Introducción: La terapia fonoaudiológica de la disfonía contempla un conjunto de recursos subjetivos cuya eficacia debe ser cuantificada.

Objetivo: En el presente trabajo se pretende cuantificar objetivamente la evolución en la calidad de la voz de pacientes disfónicos usando como referencia los índices de perturbación de la frecuencia fundamental (Jitter) e intensidad (Shimmer), y determinar si la evolución es coherente con la evaluación subjetiva del fonoaudiólogo.

Material y método: Se seleccionaron 27 pacientes disfónicos que completaron la terapia vocal entre 2009 y 2011 y que corresponden al 38\% de las consultas fonoaudiológicas por disfonía, en el Hospital de La Serena. Cada paciente, además de la evaluación subjetiva, contó con una aplicación de análisis acústico inicial y final con el programa PRAAT. Se contrastaron los resultados aplicando análisis estadístico con el software SPSS.

Resultados: Los resultados muestran que se reducen las perturbaciones de la frecuencia e intensidad registradas con el análisis acústico en la misma proporción que el grado subjetivo de la disfonía y que dichas variaciones son independientes a otras variables como la edad, sexo y tipo de disfonía.

Conclusión: Se destaca la importancia de la intervención fonoaudiológica en el tratamiento de la disfonía y la relevancia de la aplicación del análisis acústico como medio de evaluación y seguimiento objetivo.
\end{abstract}

Palabras clave: Terapia vocal, grado de disfonía, Jitter, Shimmer, análisis acústico.

\begin{abstract}
Introduction: Phonoaudiological dysphonia therapy provides a set of subjective resources whose effectiveness must be quantified.

Aim: In the present work is to objectively quantify changes in voice quality of dysphonic patients using as reference indices disturbance of the fundamental frequency
\end{abstract}

\footnotetext{
1 Fonoaudióloga. Servicio de Otorrinolaringología, Hospital de La Serena.
} 
(Jitter) and intensity (Shimmer), and determine whether the development is consistent with the subjective evaluation phonoaudiologist.

Material and method: 27 patients who completed dysphonic voice therapy between 2009 and 2011, corresponding to 38\% of consultations phonoaudiological dysphonia, at the Hospital of La Serena were selected. Each patient in addition to the subjective evaIuation, had initial and final application of the PRAAT acoustic analysis program. Results using the SPSS statistical analysis software were compared.

Results: The results show that disturbances in the frequency and intensity recorded with acoustic analysis in the same proportion will reduce the perceived degree of hoarseness and that these variations are independent of other variables such as age, sex and type of dysphonia.

Conclusion: The importance of speech therapy intervention in the treatment of dysphonia and the relevance of the application of acoustic analysis as a means of objective evaluation and monitoring is emphasized

Key words: Voice therapy, degree of dysphonia, Jitter, Shimmer, acoustic analysis.

\section{INTRODUCCIÓN}

La voz es un sonido que producido por la laringe y modificado por la acción de otras estructuras y sistemas, permiten no sólo la expresión emocional de los individuos ${ }^{1}$, sino también la expresión a través del habla ya que está a la base de gran parte de los sonidos del lenguaje.

Su producción es un fenómeno complejo ${ }^{1}$ en donde interactúan los sistemas neuromuscular, respiratorio, fonatorio, auditivo, endocrino, resonancial y articulatorio, con la coordinación de los subsistemas neurológicos central y periférico². El resultado de esta interacción proporciona las características acústicas que percibimos en la voz: intensidad, altura tonal y timbre. Cuando alguno de estos sistemas se alteran, los parámetros varían y la voz resultante cambia.

Las alteraciones de voz, llamadas disfonías, se producen cuando su realización natural se ve afectada por alguna dificultad en alguno de los niveles de producción alterando uno o varios de sus parámetros ${ }^{3}$. De esta manera, la disfonía puede constituirse como un síntoma único o bien como parte de un conjunto de síntomas de una determinada enfermedad ${ }^{2}$. La severidad de la disfonía dependerá del grado en que se hayan afectado uno 0 más de los parámetros ${ }^{2}$, y del grado de afectación en la vida de relación y en la vida profesional del paciente ${ }^{1}$.

Dada la complejidad de la naturaleza de la voz y de sus alteraciones, el tratamiento de la disfonía es multidisciplinario, contando principalmente con la acción del otorrinolaringólogo, del fonoaudiólogo, del psicólogo, entre otros.

En este contexto, el fonoaudiólogo es el encargado de valorar la funcionalidad de la voz, lo cual hace principalmente mediante mecanismos subjetivos de evaluación que dependen de la experiencia y de la experticia del profesional. También existen herramientas computacionales que permiten realizar valoraciones objetivas de la producción vocal y que permiten a su vez controlar la evolución de un determinado paciente en tratamiento.

La evaluación subjetiva, por parte del fonoaudiólogo, incluye la elaboración de una anamnesis y la aplicación de un examen funcional. Con el primero se obtienen los antecedentes de identificación del paciente, del problema, y los antecedentes psicológicos. Con el segundo se analizan un conjunto de aspectos de la producción que permiten obtener un perfil de la calidad de la voz. Entre los aspectos evaluados, se cuenta, el nivel tensional, la respiración, la intensidad, la altura tonal, timbre, ataque vocal, presencia 0 ausencia de quiebres tonales, etc. La cuantificación de la calidad de la voz puede realizarse aplicando escalas subjetivas descritas en la literatura 0 aplicando escalas de elaboración propia, de ambas maneras se obtiene una impresión global del rendimiento vocal.

Por otra parte, para realizar una evaluación objetiva se pueden utilizar diversas herramientas computacionales, entre las que se cuentan 
Dr Speech (Tiger Elemetric), SSVA (System for Single Voice Analysis), MDVP (Multi-Dimensional Voice Program), EVA (Evaluation Vocal Assistee), CSL (Computerized Speech Laboratory), PRAAT, VISHACSRE (Computerized Speech Research Environment), MEDIVOZ, etc ${ }^{4}$. Cada uno de estos programas analiza la voz grabada a través de un espectograma desde el cual se obtienen una serie de conceptos y valores que permiten diferenciar voces normales y patológicas ${ }^{5}$. Dentro de los índices obtenidos, los más utilizados en la investigación científica son los indicadores de Jitter y Shimmer. El primero es el índice de perturbación de la frecuencia fundamental (F0) ciclo a ciclo que expresado en porcentaje representa la variabilidad de ésta sin tomar en cuenta los cambios voluntarios de ella ${ }^{6}$. El umbral indicado para la normalidad en el PRAAT es de $1,040 \%{ }^{7}$, en donde los valores superiores a éste se consideran patológicos.

El segundo es el indicador de perturbación de la amplitud cuya medición sirve para cuantificar pequeños lapsos de inestabilidad de la señal vo$\mathrm{cal}^{6} \mathrm{y}$ que al igual que el primero se expresa en porcentaje. El valor de normalidad indicado para el PRAAT es de $3,08 \%{ }^{7}$. Se considera también que los valores superiores a ese promedio son indicadores de patología.

En la clínica fonoaudiológica, ambos indicadores son útiles para indicar objetivamente los cambios que se producen en la voz durante el proceso de rehabilitación de un paciente con disfonía, sin embargo, el estudio de esta variación sigue en desarrollo. Existen trabajos que indican que las perturbaciones no se reducen luego de 14 semanas de intervención, como el de Mackenzi, Millar, Sellars y Deary en $2001^{8}$, mientras que en otras investigaciones se afirma que los valores de Jitter y Shimmer se reducen luego de la terapia, estableciendo la herramienta de evaluación acústica como un indicador de éxito ${ }^{9-11}$.

La intervención fonoaudiológica en el tratamiento de la disfonía está orientada a reeducar el uso de la voz, lo cual implica un proceso de aprendizaje que se da en varias etapas en las cuales el paciente adquiere y pone en práctica el conocimiento de una adecuada técnica vocal ${ }^{12}$. Teniendo en cuenta que el objeto de la intervención carece de forma concreta, visible y palpable, el proceso de aprendizaje contempla herramientas de valoración subjetiva y está basado principalmente en aspectos sutiles en donde las sensaciones propioceptivas son la guía más importante ${ }^{13}$.

La cantidad de sesiones de la terapia de voz depende de la respuesta vocal de cada paciente, del grado y de la causa de la disfonía. Los pacientes con una disfonía de corta evolución pueden requerir de 15 a 20 sesiones, mientras que aquellos con una disfonía de larga data podrán estar en terapia durante 80 a 120 sesiones $^{13}$.

Los pacientes del Servicio de Otorrinolaringología del Hospital de La Serena que llegan derivados por disfonía deben aprender la técnica vocal para reducir el grado de disfonía y durante este proceso se utilizan diversos medios didácticos para facilitar el aprendizaje. Uno de los aspectos más relevantes en la terapia es generar en el paciente mecanismos concientes (siempre subjetivos) que le permitan evaluar en conjunto con el terapeuta los progresos obtenidos. En este sentido, la evaluación objetiva contribuye al seguimiento de su evolución, sin embargo, la descripción de la variabilidad esperada de sus indicadores en el contexto terapéutico ha sido poco descrita.

\section{OBJETIVO}

En el presente trabajo se pretende cuantificar objetivamente la evolución en la calidad de la voz de pacientes disfónicos usando como referencia los índices de perturbación de la frecuencia fundamental (Jitter) e intensidad (Shimmer) obtenidos con el software de análisis acústico PRAAT, y determinar si la evolución es positiva y si ésta es coherente con la evaluación subjetiva del profesional fonoaudiólogo.

\section{MATERIAL Y MÉTODO}

Se seleccionaron 27 pacientes disfónicos del Servicio de Otorrinolaringología del Hospital de La Serena que ingresaron a tratamiento fonoaudiológico entre 2009 y 2011 que equivalen al $38 \%$ del total de las consultas por disfonía en ese período. Los sujetos seleccionados contaron con las siguientes características: 
1. Tener diagnóstico otorrinolaringológico de disfonía.

2. Presentar una evaluación fonoaudiológica subjetiva y objetiva al principio de la terapia y al término de ésta.

3. Haber cumplido con todas las etapas relacionadas con el desarrollo de la técnica vocal.

4. No presentar disfonía de origen psicógeno.

El grupo de pacientes seleccionados estaba constituido por 17 adultos y 10 niños. Las edades de los adultos variaban entre los 18 y los 74 años, mientras que la de los niños variaba entre 8 y 13 años. El grupo de adultos lo conformaban 7 hombres y 10 mujeres y en el grupo infantil eran todos varones.

En el grupo de adultos varones, se registraron 2 pacientes con disfonía músculo tensional, 2 con nódulos cordales bilaterales, 3 con parálisis cordal, 1 presentó además reflujo faringolaríngeo y otro reflujo gastroesofágico. En el caso de las mujeres, 6 registraban diagnóstico de disfonía músculo tensional, 1 nódulos cordales bilaterales y 4 presentaban reflujo faringolaríngeo como antecedente. En el caso de los niños, 9 presentaban diagnóstico de nódulos cordales bilaterales y 1 paciente tenía el diagnóstico de disfonía espástica, 1 paciente registró reflujo gastroesofágico y 2 presentaban asma bronquial. Cabe señalar que sólo en el grupo de mujeres se observó además antecedentes de depresión en 4 de ellas y estrés en 5 .

Cada sujeto fue evaluado individualmente en una sala del Servicio de ORL del Hospital de La Serena no insonorizada, pero silenciosa. Se realizaron 2 grabaciones por paciente, una de ellas durante la evaluación inicial y la otra al término del proceso terapéutico. Se utilizó un micrófono cuya frecuencia de respuesta está entre 80 y 16.000 hz. El paciente debía emitir una /a/ sostenida por al menos 3 segundos usando el micrófono a $15 \mathrm{~cm}$ y a un ángulo de $45^{\circ}$. El análisis acústico se realizó con el software PRAAT con el cual se obtuvo los valores para Jitter (perturbación de la frecuencia) y Shimmer (perturbación de la intensidad) del tramo medio del sonograma.

La evaluación fonoaudiológica, realizada por un solo examinador, incluyó además la graduación subjetiva de la disfonía, es decir, el grado general del desvío de la voz, la que podría darse en tres niveles: grado 1 (leve) para alteraciones vocales discretas, grado 2 (moderado) para alteraciones evidentes y grado 3 (severo) para alteraciones vocales intensas.

Luego de la evaluación inicial se comenzó la terapia vocal una vez por semana con una duración aproximada de 45 minutos. A cada uno de los pacientes se le aplicó un programa de actividades agrupadas en 7 unidades, las cuales se adaptaron a los distintos requerimientos que puede presentar un paciente dependiendo del grado y de las causas de la disfonía:

1. Relajación

2. Respiración

3. Técnica de resonancia

4. Suavización del ataque vocal

5. Tono vocal

6. Intensidad

7. Integración de parámetros vocales

Si bien el programa de intervención presentó variaciones en su aplicación, cada una de estas variaciones estaban orientadas a conseguir una voz estable, sin tensiones y de intensidad, tono y timbre adecuados, es decir, orientadas a que el paciente aprenda y aplique la técnica vocal en la voz hablada.

El término de la terapia se decretó una vez que el paciente logró los objetivos de cada unidad y después de haber ejercitado las condiciones ideales de producción de la voz hablada. La duración general del programa estaba condicionada por la asistencia, la ejercitación en el hogar y las condiciones de salud generales (resfríos, alergias, etc.), todos antecedentes registrados en la base de datos.

Los resultados de las evaluaciones iniciales y finales se compararon a través del análisis estadístico de T de Student con el apoyo del software SPSS.

\section{RESULTADOS}

En la evaluación inicial se registró para el índice Jitter un promedio de 1,09, una desviación típica de 0,86 , y un valor mínimos de 3,81 y un máximo de 0,29 . En la evaluación final el promedio de este indicador fue de 0,54, con una desviación típica de 
0,60, un valor mínimo de 0,18 y un valor máximo de 2,87. La diferencia existente entre ambos promedios fue de 0,55 , indicando una reducción de la perturbación de la frecuencia (Jitter) en el $50 \%$ (ver Tabla 1 y Figura 1).

El primer registro del índice Shimmer corresponde a 6,92 como promedio, con una desviación típica de 4,14, un valor mínimo de 1,30 y un máximo de 18,92. En el registro final se obtuvo un promedio de 3,43, una desviación típica de 1,34, un valor mínimo de 1,30 y un máximo de 5,88. La variación entre ambos promedios fue de 3,49 evidenciando una reducción de $50 \%$ en la perturbación de la intensidad (Shimmer) (ver Tabla 2 y Figura 2).

La aplicación de la prueba T de Student muestra una variación estadísticamente significativa entre la evaluación inicial para los índices Jitter y

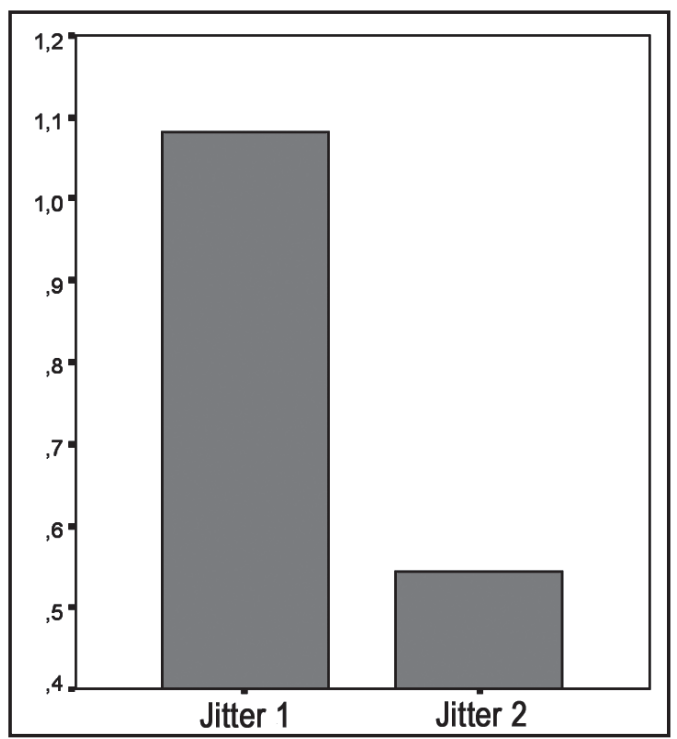

Figura 1. Comparación Jitter 10 inicial, y Jitter 20 final.
Shimmer, y la evaluación final de los mismos, pues el valor $p$ es inferior a 0,05 (ver Tabla 3).

El promedio del grado de disfonía resultante de la evaluación subjetiva inicial fue de 2 (moderado), registrándose pacientes con disfonía en grado 1 (leve) como valor mínimo y en grado 3 (severo) como máximo. Al término de la terapia se observó un promedio del grado disfonía de 1 (leve) siendo este mismo el valor máximo, mientras que el mínimo fue de 0 , es decir, ausencia de disfonía. La reducción del grado de disfonía observado fue del $50 \%$ ) (ver Tabla 4 y Figura 3 ).

Las características de los sujetos seleccionados para el presente estudio, fueron diversas en cuanto a edad, sexo y tipo de disfonía, por lo que los resultados de perturbación de Jitter y Shimmer caso a caso fueron también variados, en relación a estas condiciones. Esto se puede apreciar en las Tablas 1 y 2 al observar los valores mínimos y máximos. Sin embargo y teniendo esto en cuenta este escenario, es posible afirmar que del grupo total de los sujetos, el 93\% registró una disminución de la perturbación de la frecuencia fundamental, el $85 \%$ de los pacientes redujo la perturbación de la intensidad registrada en el análisis acústico. Por otro lado el $100 \%$ mejoró la cualidad de la voz representada por el grado de disfonía, aunque no todos los pacientes consiguieron una reducción total. Todas las variaciones registradas fueron estadísticamente significativas de acuerdo al análisis con la prueba T de Student del software SPSS (ver Tablas 3 y 5 ).

Las pruebas estadísticas aplicadas indican que la variación, o más bien la reducción de los índices Jitter y Shimmer, y del grado de disfonía fue independiente del sexo, de la edad y del tipo de disfonía. Tampoco se observaron relaciones significativas entre esta reducción y el tiempo de tratamiento, la asistencia a la terapia y la presencia de factores psicógenos como el estrés o depresión.

Tabla 1. Resultados medición de Jitter al inicio de la teriapia, posterapia y grado de variación

\begin{tabular}{|l|c|c|c|c|}
\hline Índice & Promedio & Desviación estándar & Valor mínimo & Valor máximo \\
\hline Jitter inicial & 1,09 & 0,86 & 0,29 & 3,81 \\
\hline Jitter final & 0,54 & 0,60 & 0,18 & 2,87 \\
\hline Variación Jitter inicial y final & $0,55=50 \%$ & & \\
\hline
\end{tabular}


Tabla 2. Resultados medición de Shimmer al inicio de la terapia y posterapia

\begin{tabular}{|l|c|c|c|c|}
\hline Índice & Promedio & Desviación estándar & Valor mínimo & Valor máximo \\
\hline Shimmer inicial & 6,92 & 4,14 & 1,30 & 18,92 \\
\hline Shimmer final & 3,43 & 1,34 & 1,30 & 5,88 \\
\hline Variación Shimmer inicial y final & $3,49=50 \%$ & & \\
\hline
\end{tabular}

\section{DISCUSIÓN}

La intervención fonoaudiológica en la disfonía se basa en principios subjetivos que giran en torno a sensaciones propioceptivas que cada paciente debe desarrollar para poder ejecutar concientemente cambios en la manera de producir la voz que lo lleva a obtener un mejor rendimiento vocal desde el punto de vista fisiológico y acústico. La aplicación de principios terapéuticos como el de la adecuada relajación, respiración e impostación de la voz resulta variable en cada caso, ya que se debe responder a las necesidades cognitivas y socioafectivas de cada sujeto, lo que lleva a interpretar la implementación terapéutica como un hacer dinámico, flexible y con un carácter subjetivo a la hora de evaluar los procedimientos realizados.

Como un intento de objetivizar y unificar criterios tanto de evaluación como de intervención, se ha incorporado a la clínica fonoaudiológica el uso de análisis acústico con softwares computacionales que han permitido generar perfiles acústicos de voces tanto normales como patológicas. En el contexto de las disfonías, las investigaciones realizadas establecen que los indicadores posibles

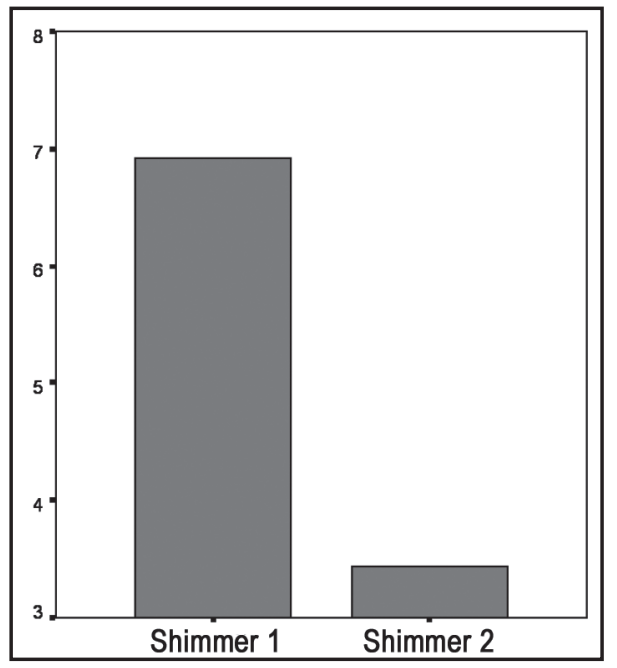

Figura 2. Comparación Shimmer 10 inicial y Shimmer 20 final.

de obtener van a ser siempre más altos en las disfonías en contraste con los registros de las voces normales. Sin embargo, falta claridad para determinar en qué momento exacto cierto valor es indicador de disfonía o cuánto es lo que éstos pueden 0 deben variar para determinar el término

Tabla 3. Resultados de la prueba T de Student proporcionado por el software Spss. Aquí se evidencian las diferencias significativas entre las evaluaciones iníciales y finales

\begin{tabular}{|c|c|c|c|c|c|c|c|c|}
\hline & \multicolumn{8}{|c|}{ Diferencias relacionadas } \\
\hline & \multirow[t]{2}{*}{ Media } & \multirow[t]{2}{*}{$\begin{array}{c}\text { Desviación } \\
\text { típ. }\end{array}$} & \multirow[t]{2}{*}{$\begin{array}{l}\text { Error típ. de } \\
\text { la media }\end{array}$} & \multicolumn{2}{|c|}{$\begin{array}{l}\text { 95\% Intervalo de confianza } \\
\text { para la diferencia }\end{array}$} & \multirow[t]{2}{*}{$\mathrm{t}$} & \multirow[t]{2}{*}{$\mathrm{gl}$} & \multirow{2}{*}{$\frac{\text { Sig. (bilateral) }}{\text { p-valor }}$} \\
\hline & & & & Inferior & Superior & & & \\
\hline $\begin{array}{rr}\text { Par } 1 & \text { JITTER1 } \\
& \text { JITTER2 }\end{array}$ &, 5404 & ,63636 & ,12247 & ,2886 & ,7921 & 4,412 & 26 & 000 \\
\hline $\begin{array}{rr}\text { Par } 2 & \text { SHIMER1 } \\
& \text { SHIMER2 }\end{array}$ & 3,4870 & 3,55933 & ,68499 & 2,0790 & 4,8951 & 5,091 & 26 & 000 \\
\hline
\end{tabular}

